\title{
Optimization Design of Printed Circuit Board Structure Based on Modal Analysis
}

\author{
Zhang Weifen ${ }^{1, a}$, Li Yongmei ${ }^{2, b}$, Qian $\mathrm{Ru}^{3, \mathrm{c}}$ \\ ${ }^{1}$ Department of Mechanical Engineering,Chengxian Institute,Southeast University,Nanjing , \\ 210088, China \\ ${ }^{2}$ Department of Mechanical Engineering,Chengxian Institute,Southeast University,Nanjing , \\ 210088,China \\ ${ }^{3}$ Department of Mechanical Engineering, Chengxian Institute, Southeast University,Nanjing , \\ 210088,China \\ aemail:11376714@qq.com, bemail:149687158@qq.com, ${ }^{\mathrm{c} e m a i l}$ :715216392@qq.com
}

Keywords: PCB;modal analysis;optimized design of structure;natural frequency

\begin{abstract}
According to the problems of the vibration reliability of pcb in practical, a finite element model was established according to PCB actual parameters,and their first 4-order natural frequencies and modal modes are obtained by modal analysis, and the finite element model which is also helpful for the structural improvement is proved to be correct through the experimental modal analysis. And based on these,optimized design of PCB structure is accomplished for increasing the lower rank natural frequencies of pcb and enhancing the ability of anti-vibration and shock of pcb.
\end{abstract}

\section{Introduction}

Accompanied by the widely used of electronic devices, Printed Circuit Board (PCB) has become the most important component of electronic equipments. Due to the limitation of major, circuit designers generally do not consider the vibration reliability of boards. Nevertheless, with the development of PCB in the directions of high accuracy, small aperture, small spacing, multilayer, high speed transmission ${ }^{[1]}$, higher demand of reliability was put forward on PCB. The dynamic performance of PCB is the key factor to ensure the electronic device working reliable, especially in the environment of vibration. Therefore, researching and reforming dynamic performance of PCB has significance for whether the device running normally or not.

\section{Theoretical modal analysis of PCB}

Figure 1 is the diagram of PCB. Considering the length after loading while model analyzing, its effective length is $160 \mathrm{~mm}$ and effective width is $95 \mathrm{~mm}$. The thickness of epoxy resin board is $1.5 \mathrm{~mm}$, and the reinforcing aluminum plate is $1.0 \mathrm{~mm}$ thick. Two short-length opposite edges of board are fixed in the case by wedge shaped locking bar, and the lower edge is inserted into the motherboard. Through analyzing PCB model by ANSYS, the resonant frequency and the type of vibration could be figured out. The various material parameters in PCB are shown in table 1.

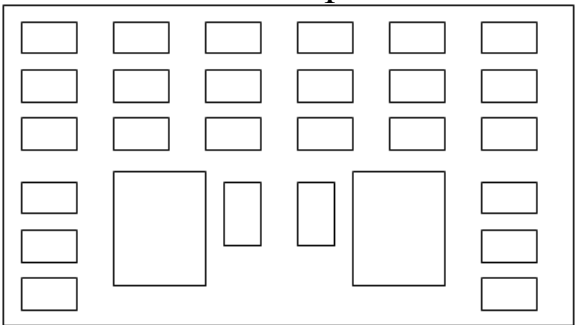

Fig.1. Diagram of PCB 
Table 1 Material parameters in PCB

\begin{tabular}{|c|c|c|c|}
\hline material & $\begin{array}{c}\text { elastic } \\
\text { modulus } / \mathrm{GPa}\end{array}$ & density / $\left(\mathrm{kg} . \mathrm{m}^{3}\right)$ & Poisson ratio \\
\hline PCB & 22 & 1850 & 0.28 \\
\hline microchip & 16 & 2420 & 0.30 \\
\hline aluminum plate & 65 & 2700 & 0.2 \\
\hline copper & 120 & 8940 & 0.35 \\
\hline
\end{tabular}

Establishing model is the key step in the whole process of finite element analysis. The quality of the finite element model influences the calculation accuracy directly. In this paper, the models of whole board, additional components and modules are directly build by finite element method. According to the size of the components, the printed board is divided into different integrated forming regions ${ }^{[2]}$. And the solid45 element is selected for meshing. The finite element model is shown as figure 2 .

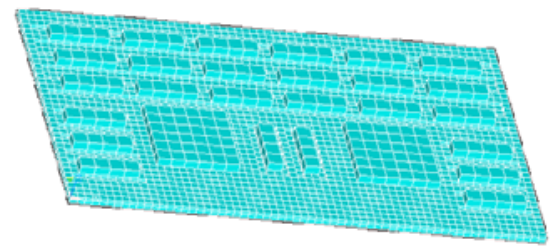

Fig.2. The finite element model after meshing

Through applying ANSYS model analysis module, the natural frequency in first four stages of target board were extracted by using Block Lanczos method, as shown in figure 3. The types of vibration are shown in figure 4, it appears that the PCB has large deformation in its middle part. If microchips which were set up there have poor reliability, they would influence the whole PCB's performance.

\begin{tabular}{|ccccc|}
\hline Set & Frequency & Load Step & Substep & Cumulative \\
\hline 1 & 264.28 & 1 & 1 & 1 \\
2 & 488.50 & 1 & 2 & 2 \\
3 & 686.47 & 1 & 3 & 3 \\
4 & 1085.5 & 1 & 4 & 4 \\
\hline
\end{tabular}

Fig.3. Natural frequency of PCB

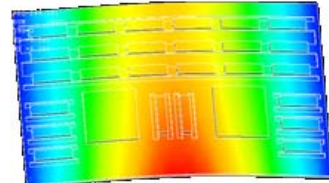

a) first stage

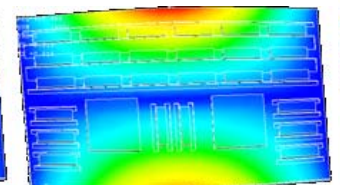

b) second stage

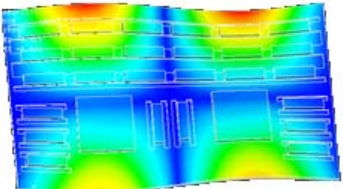

c) third stage

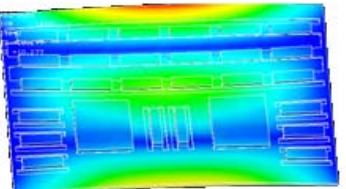

d) forth stage

Fig.4. Vibration types of PCB in first four stage

\section{Experimental modal analysis}

In order to confirm the result of theoretical modal analysis, experimental modal analysis was carried out. The experiment was operated by the way of hanging freely by rubber band for seeking the natural frequency of PCB. The instruments used in this trial mainly include SignalCalc 620 dynamic signal analyzer,B\&K4374 acceleration sensor,B\&K2692 charge amplifier, MEGGITT ENDEVCO 2302-10 impact test hammer and modal analysis software. Experimental principle together with connection between various instruments are shown in the figure 5 . 


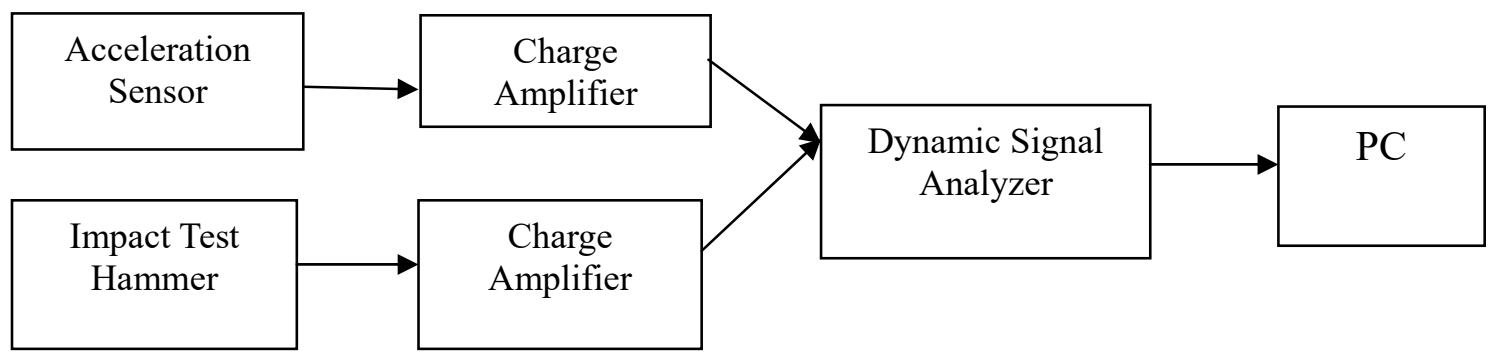

Fig.5. Block diagram of experimental modal test system

Owing to the dense microchips on the target board, the number of optional knocking points or test points were limited. Therefore, this experiment used single point excitation method, and selected only one test point. In this trial, sensors were stuck onto specimen by glue whose stiffness is perfect. Then turn on the signal collector, afterwards, knocked the test point on the specimen by hammer. Finally, after the dynamic signal analyzer handled the input signals and response signals, the results of the model information such as frequency response functions of the system would be displayed in the model analysis software on PC.

Pay particular attention to the setup of sensors while testing:

(1)Avoid arranging the sensors on the modal nodes or knocking multiple points. Comparing with the signals could analysis the resonance frequency of the target board in each stage. Otherwise, if the sensors are attached to the places where the nodes are, it will generate the phenomenon of loss frequency.

(2)Sensors shall be setup on the back of the basilar plate and so does the point where the impulse hammer knocks. If they are located on the same side of reinforcing aluminum plate, it will probably produce the results which are not the model signals of PCB to be analyzed. And if they are situated on both sides, the model signals are likely influenced by the transfer rate between PCB and aluminum board.

(3)For the sake of generating signals with broader spectrum, hammerhead chooses the material of high hardness alloy steel.

(4)In order to avoid signals far away from the true value owing to too few samples, and to avert signals beyond the main wave in the resolution range because of too many samples which leads to the unreliability of high frequency part of transfer function, the sampling interval of signal acquisition device is $195.313 \mu \mathrm{s}$.

(5)The sensitivity of impulse hammer is $2.27 \mathrm{mv} / \mathrm{N}$ in this experiment.

The results of comparing natural frequency achieved by experiment with which are obtained by model analysis is shown in table 2 .

Table 2 Comparison between finite element analysis and experimental results of natural frequency

\begin{tabular}{|c|c|c|c|c|}
\hline stage & 1 & 2 & 3 & 4 \\
\hline $\begin{array}{c}\text { calculated value } \\
\text { frequency }\end{array}$ & 264.28 & 488.50 & 686.47 & 1085.5 \\
\hline test value (Hz) & 260.36 & 460.57 & 640.69 & 970.94 \\
\hline error (\%) & 1.48 & 5.71 & 6.67 & 10.56 \\
\hline
\end{tabular}

As is shown in the chart, there are several differences between calculated values and test results. The reasons for this are as follows:

(1)Finite element model is a simplified model. The shape of microchips and the mode of coupling were simplified for the sake of all the common nodes of reinforcing aluminum plate and PCB could be coupled well. So the geometry dimensions and the actual sizes are not exactly the same, in other 
words, it has error compared with actual structure.

(2)The error existing among the loads which act on target board for calculation or test board could lead to the generation of error.

(3)In the manufacturing process of the test board, the various handling make the characteristics of the materials have a certain difference. However, materials are considered as homogeneous objects while calculating.

(4)The error caused by testing principle or signal-to-noise ratio make the analysis results not very accurate and also have the possibility to miss some modes.

But from the point of view of natural frequency, the relative error between finite element modal analysis and experimental model analysis is small enough to be within the error range. Thus, the finite element model is substantially correct which could provide references for structure modification of PCB.

\section{Structural optimization design of PCB}

Due to the PCB will be arranged in the chassis, in order to improve its reliability, the low order natural frequency of PCB must be much higher than chassis's which is able to avoid the resonance of them. The method of improving the low order natural frequency of PCB includes arranging reinforcing bar or plate on the board, increasing the number of fastening points ${ }^{[3][4]}$, changing the size or the material of the circuit board and installing locking strips. The observation of the reinforcing aluminum plate found that the upper part of the plate has a transverse groove due to the need for its installation as shown in figure 6. Although it is not a slot, the rigid structure of the reinforcing aluminum plate is damaged and the low order frequency of whole board is getting lower because the actual load is added to the inner side of the wedge strip, which is very close to the end of the groove while installing, so, it is necessary to optimize the shape of the aluminum plate.

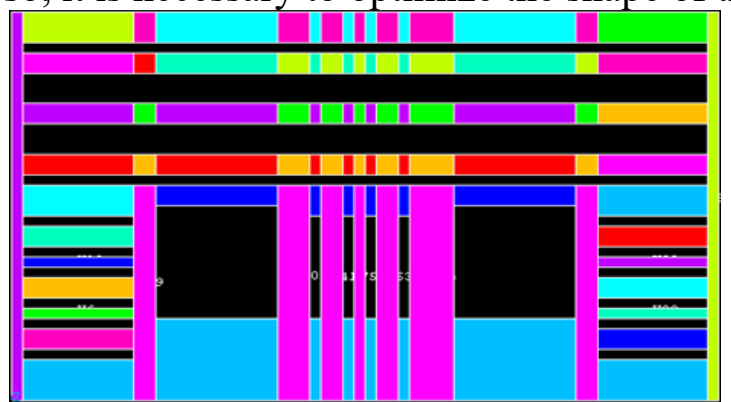

Fig.6. The shape of the reinforcing aluminum plate

Optimizing the reinforcing aluminum plate in order to obtaining the minimum value of the natural frequency in a certain reduction of volume. The command stream program is as follows:

/TITLE, tuo pu you hua

/REPLOT

/COM, Structural

ET,1,SOLID95

MPDATA,EX,1,,6e10

MPDATA,PRXY,1,0.1

MPDATA,DENS, 1,2700

BLC4,0,0,0.16,0.095,0.001

FLST,5, 12,4,ORDE, 2

MSHAPE,0,3D 


\section{FINISH}

/SOLU

/GO

DA,P51X,ALL,

\section{SAVE}

allsel

finish

tofreq,freq1,sing, 1

tovar,freq1,obj

tovar,volume,con, 0.000008 , actual

totype,oc

todef, 0.00001

toloop, 40,1

\section{FINISH}

After executed the eighth iteration, the first order frequency reached the maximum under the circumstance that the volume was reduced to $8 \mathrm{e}-6 \mathrm{~m}$. The topology optimization of the reinforcing aluminum plate is shown in figure 7.

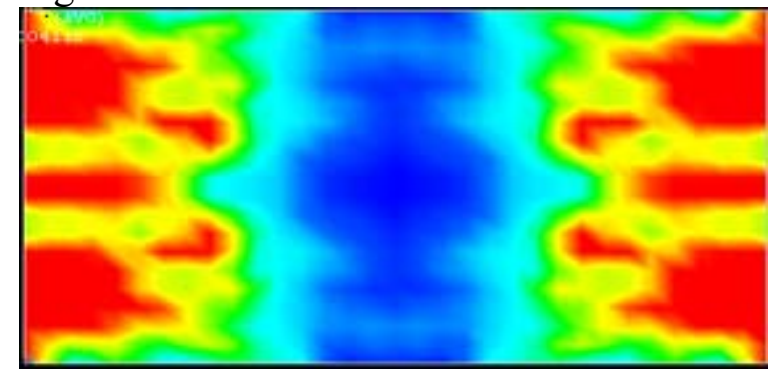

Fig.7. Topology optimition of the reinforcing aluminum plate

As shown in figure 7, blue represents the area which pseudo density is close to zero and can be discarded, however, red is a region with a pseudo density close to one which has sensitive stiffness and must be preserved in design. The area near the two short sides of the target board is the most sensitive area of stiffness, yet the groove on the top of the board ruins the rigid structure, so the reinforcing plate doesn't work well actually. Besides, the structure and the constraint condition of reinforcing plate is similar to the substrate's, thus, figure 7 is also suitable for the structural design of the substrate, it means do not set too large or too many holes in the position which is sensitive to stiffness while arranging microchips or through-holes. At the same time, the topology optimition figure above indicates that it will have a good effect if reinforcing bars can be arranged in the area which has big pseudo density.

Based on the analysis above, the distance of the groove and constrained edge is broadened $0.010 \mathrm{~m}$ while modeling as shown in figure 8 . Carrying out the modal analysis of the target plate again, the first order natural frequency is increased by $50 \mathrm{~Hz}$. 


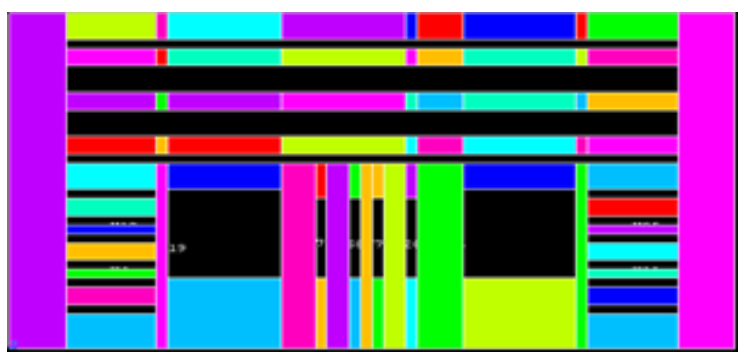

Fig.8. structure improvement of reinforcing plate

Moreover, the materials and the shapes of the reinforcing aluminum and the PCB shall be considered synthetically. Do not arrange large holes in the sensitive areas of stiffness but put some reinforcing bars there. The microchips which are sensitive to stress and strain should be as far as possible set in the area which has small stress and strain. In order to avoiding the resonance of the chips and the circuit board, the circuit board can be packaged by using material such as silicon rubber as a whole ${ }^{[5]}$.

\section{Conclusion}

This article analyzed the target board by using finite element software and experimental modal analysis and carriedout the topology optimization design of aluminum plate for higher low order natural frequency of $\mathrm{PCB}$, and of course, successfully improved the ability of anti vibration or impact of PCB, consequently improved the reliability of the whole electronic equipment. In addition, it is important to attend to the influence of installation conditions on base frequency of the circuit board which demands high reliability of vibration. When designing, it ought to use spring shims and locking bars as locking devices. The vibration of chassis should be also taken into account to avoid the resonance of circuit board and chassis which reduces the vibration reliability of PCB. At last, the circuit demands high reliability of vibration could be strengthened by using titanium alloy or use viscoelastic material to reduce vibration.

\section{References}

[1]Chen Shi-xu,Jing Shou-zhao.Anti-vibration Design of PCB's Placement and Routing[J].Electronics Quality, 2004, (6):16-18.

[2] Wang Hong-fang, Zhao Mei.A Study on The Dynamic Behavior of A Printed Circuit Board in Electronic Box for Ship Application[J].Journal of Vibration and Shock,2000,19 (1): 49-51.

[3] Q.J.Yang.Experimental Modal Analysis of PBGA Printed Circuit Board Assembles[J].Proc IEEE Paper 07803-4157-0/97,1997,290-293.

[4] Pitarrsi J M,Di Edwardo A V. A Design Approach for the Systematic Improvement of Support Location for Vibrating Circuit Cards. ASME J.Electron.Package,1993,115(1):118-123.

[5] XU Zhao-mei.Dynamic properties analysis of SMT printed circuit board assembly[J].Electronic Components and Materials,2010,12 (29): 56-58. 